\title{
MULTI-CRITERIA DECISION MAKING IN THE PRESENCE OF IGNORANCE USING THE DS/AHP METHOD
}

\author{
Nadezhda I. Nedashkovskaya* \\ Institute for Applied System Analysis, National Technical University of Ukraine "Kiev Polytechnic \\ Institute", Kiev, Ukraine \\ E-mail: n.nedashkivska@gmail.com
}

\begin{abstract}
The paper describes different cases of rank reversals in the DS/AHP method. These cases deal with the rank reversals when an irrelevant decision alternative that is dominated by one or more previously existing decision alternatives is added or removed. Two conditions for having rank reversals when the DS/AHP method is used to solve MCDM problems are defined. The first condition deals with the change in belief measures of groups of decision alternatives. The second one concerns the comparison of belief intervals.
\end{abstract}

Keywords: multi-criteria decision making, ignorance, DS/AHP, rank reversals, Dempster's rule of combination

\section{Introduction}

In the past decades, the commonly used Analytic Hierarchy Process (AHP) (Saaty 1988, 2003) for solving multi-criteria decision making (MCDM) problems, which is based on expert preference judgements, has been considerably extended and modified. One of its extensions is the DS/AHP method, which incorporates the AHP method with the Dempster-Shafer theory of evidence (Beynon, 2002; Hua et al., 2008; Nedashkovskaya, 2010).

In comparison to the standard AHP methods, use of DST allows a greater level of control over judgements made by a decision maker. While solving problems of practical importance, a decision maker is not always able to make pairwise comparisons between all decision alternatives. However, this is a prerequisite for applications of the AHP method and almost all of its modifications. For a practical MCDM problem, information about decision alternatives may be incomplete due to time limitations, ignorance, intangible nature of some attributes, limited information processing capabilities (Forman, 1987), etc. Most of the researchers solve a MCDM problem with incomplete information by the following two-stage procedure. At the first stage, an MCDM problem with complete information is constructed by complementing missing values of decision matrices through a learning mechanism (Fortes et al., 2006) and heuristic rules (Quinten and Raaijmakers, 1999). At the second stage, usual MCDM methods are applied to solve the problem with complete information. Unlike most of the current methods, the DS/AHP method solves a problem directly based on its incomplete decision matrix.

One of the problems associated with the use of MCDM techniques is a possibility to change a ranking of decision alternatives when an alternative is added or deleted. This phenomenon is known as rank reversal. A detailed discussion on different types of rank reversals in the AHP method will be found in the literature of the subject (Barzilai and Lootsma, 1994; Dyer, 1990; Forman, 1987; Nedashkovskaya, 2005; Triantaphyllou, 2001; Saaty, 1990, 2006).

For instance, a debate has been ongoing between practitioners of the AHP and the MAUT methods about whether the ranking of decision alternatives should be allowed to change when an "irrelevant"

\footnotetext{
${ }^{*}$ Corresponding author
} 
alternative, i.e. an alternative that is dominated by one or more previously existing alternatives, is added to the set of decision alternatives. The MAUT method is based on axioms that do not allow rank reversals to occur when an irrelevant alternative is introduced for consideration (Luce and Raiffa, 1957). On the other hand, in so called "closed" systems with a fixed amount of resources rank reversals are not only allowed but are often desirable (Saaty, 1990; Forman, 1987). Quite the contrary, when dealing with "open" systems where resources can be added or removed, any rank adjustments should be precluded. Distributive synthesis is recommended for the closed systems, and ideal synthesis - for the open systems (Saaty, 1990). However, further investigations revealed that rank reversals also occur when ideal synthesis is used (Barzilai and Lootsma, 1994; Triantaphyllou 2001; Nedashkovskaya, 2005; Saaty, 2006).

More recently, three kinds of relations among alternatives are being discussed: independence, conditional independence and functional dependence (Saaty, 2006). It was observed that a ratio of priorities of alternatives is invariant to adding a new alternative when priorities of criteria depend on the alternatives. This invariance should also hold in the stronger case when criteria are independent of alternatives, but alternatives themselves are structurally independent of one another. When proportionality is not maintained because of structural dependence for each criterion, rank can reverse.

In this paper two conditions for having rank reversals when the DS/AHP method is used to solve MCDM problems are defined. The first condition deals with the change in belief measures of groups of decision alternatives. The second one concerns the comparison of belief intervals.

The paper is organized as follows: the DS/AHP method is briefly described in Section 2; conditions and cases of rank reversals are introduced in Section 3; several examples of rank reversals in the DS/AHP method are given in Section 4; a concluding remark is presented in Section 5.

\section{The DS/AHP method}

To combine aspects of DST and AHP the DS/AHP method of MCDM is introduced (Beynon et al., 2000). DS/AHP (as in AHP) is based on a hierarchical (decision tree) structure and consists of several steps (Beynon, 2000, 2002; Hua et al., 2008; Nedashkovskaya, 2010):

1) to find criteria priority values (CPVs) using the standard eigenvector method of AHP;

2) to identify groups of DAs for each criterion. The number of groups identified is decided by the decision maker (DM) and may reflect the amount of knowledge the DM has on the criterion. Within one group, DAs have equal favourability to the frame of discernment $\Theta$;

3 ) to construct the specific criteria knowledge matrix for each criterion (see later), where its values are the measures of favourability of the groups of DAs with respect to $\Theta$;

4) to calculate the associated sets of priority values for the criteria knowledge matrices (bpa values for groups of DAs and $\Theta$ ) again using the standard eigenvector method of the AHP. Analytic functions are constructed to find these criteria bpa values (Beynon, 2002);

5) to aggregate the criteria bpa values into a single bpa $m_{\text {aggr }}$ using Dempster's rule of combination:

$$
\begin{aligned}
& m_{1} \oplus m_{2}(A)=\frac{1}{K} \sum_{X \cap Y=A} m_{1}(X) m_{2}(Y) \\
& K=\sum_{X \cap Y \neq \varnothing} m_{1}(X) m_{2}(Y)=1-\sum_{X \cap Y=\varnothing} m_{1}(X) m_{2}(Y)
\end{aligned}
$$

The resulting bpa values are the amounts of exact belief in groups of DAs based on the combined evidence from all criteria. Specifically, $m_{\text {aggr }}(\Theta)$ is the value of combined uncertainty;

6) to find levels of belief $(\mathrm{Bel})$ and plausibility $(\mathrm{Pls})$ and obtain the belief interval $[\mathrm{Bel}(\cdot), P l s(\cdot)]$ for each group of DAs and $\Theta$. 


\section{Conditions and cases of rank reversals in the DS/AHP method}

Suppose $n$ DAs $A_{1}, A_{2}, \ldots, A_{n}$ are evaluated in terms of two decision criteria $C_{1}$ and $C_{2}$. For these criteria $d_{1}$ and $d_{2}$ groups of DAs $S_{11}, S_{12}, \ldots, S_{1 d_{1}}$ and $S_{21}, S_{22}, \ldots, S_{2 d_{2}}$, respectively, are identified as being comparable to the frame of discernment $\Theta$, where $S_{1 k} \cap S_{1 l}=\varnothing, S_{2 p} \cap S_{2 r}=\varnothing$, $k, l=1, \ldots, d_{1}, p, r=1, \ldots, d_{2}$. Using the DS/AHP method, the belief measures $\operatorname{Bel}(\cdot)$ and belief intervals $[\operatorname{Bel}(\cdot), P l s(\cdot)]$ are calculated for each group of DAs and the frame $\Theta$.

We are interested in the conditions of changes in the DA ranking orders with the use of DS/AHP method when a DA is added to the set of DAs. In this paper two sets of such conditions are defined. The first set of conditions deals with the changes in the belief-based ranking orders of DAs. Belief measures $\operatorname{Bel}\left(S_{i}\right)$ and $\mathrm{Bel}^{*}\left(S_{i}\right)$ denote the amounts of exact belief in a group $S_{i}$ based on combined evidence from two criteria before and after a DA is added to the set of DAs, respectively.

Condition 1: Rank reversal appears if belief in a group $S_{i}$ exceeds belief in a group $S_{j}$ before a DA is added to the set of DAs and belief in a group $S_{i}$ becomes less than belief in a group $S_{j}$ after it is added, i.e. $\operatorname{Bel}\left(S_{i}\right)>\operatorname{Bel}\left(S_{j}\right)$ and $\operatorname{Bel}^{*}\left(S_{i}\right)<\operatorname{Bel}^{*}\left(S_{j}\right)$. Rank reversal also appears, if $\operatorname{Bel}\left(S_{i}\right)<\operatorname{Bel}\left(S_{j}\right)$ and $\operatorname{Bel}^{*}\left(S_{i}\right)>\operatorname{Bel}^{*}\left(S_{j}\right)$ or if the belief measures for groups $S_{i}$ and $S_{j}$ have coincided (differed) before a DA is added to the set of DAs and become different (similar) after it is added. Thus, the overall condition 1 of rank reversal is as follows:

$$
\begin{aligned}
& \left(\Delta \operatorname{Bel}_{i j} \Delta B e l_{i j}^{*}<0\right) \vee\left(\Delta B e l_{i j}=0\right) \wedge\left(\Delta B e l_{i j}^{*} \neq 0\right) \vee\left(\Delta B e l_{i j} \neq 0\right) \wedge\left(\Delta B e l_{i j}^{*}=0\right), \\
& \Delta \operatorname{Bel}_{i j}=\operatorname{Bel}\left(S_{i}\right)-\operatorname{Bel}\left(S_{j}\right), \Delta B e l_{i j}^{*}=\operatorname{Bel}^{*}\left(S_{i}\right)-\operatorname{Bel}^{*}\left(S_{j}\right) .
\end{aligned}
$$

The second set of conditions of changes in the DA ranking orders concerns the comparison of belief intervals $\left[\operatorname{Bel}\left(S_{i}\right), \operatorname{Pls}\left(S_{i}\right)\right]$ and $\left[\operatorname{Bel}^{*}\left(S_{i}\right), P l s^{*}\left(S_{i}\right)\right]$, where plausibility measures $\operatorname{Pls}\left(S_{i}\right)$ and $P l s^{*}\left(S_{i}\right)$ denote the maximum probability of possible support to a group $S_{i}$ before and after a DA is added to the set of DAs, respectively.

Condition 2: Rank reversal appears, if preference relation defined by belief intervals for groups $S_{i}$ and $S_{j}$ changes after a DA is added to the set of DAs.

To obtain the preference relations among groups of DAs, we need a mechanism to generate the rank of groups of DAs based on their belief intervals. One of the methods is the evidential reasoning ranking method (Wang et al., 2006). Let us take a brief look at this method. Lets denote by $\left[\operatorname{Bel}\left(S_{i}\right), \operatorname{Pls}\left(S_{i}\right)\right]$ and $\left[\operatorname{Bel}\left(S_{j}\right), \operatorname{Pls}\left(S_{j}\right)\right]$ the belief intervals of groups $S_{i}$ and $S_{j}$, respectively. If $\operatorname{Bel}\left(S_{i}\right)>\operatorname{Bel}\left(S_{j}\right)$ and $\operatorname{Pls}\left(S_{i}\right)>\operatorname{Pls}\left(S_{j}\right)$, based on interpretation of the belief interval (see Figure 1), group $S_{i}$, compared with group $S_{j}$, will have a higher probability of exact support and a lower probability of refusal. In such case, $S_{i}$ is said to be preferred to $S_{j}$. For two general belief intervals, the degree of preference of $S_{i}$ over $S_{j}$, denoted by $P\left(S_{i}>S_{j}\right) \in[0,1]$, is defined as follows (see also Hua et al., 2008):

$$
P\left(S_{i}>S_{j}\right)=\frac{\max \left[0, \operatorname{Pls}\left(S_{i}\right)-\operatorname{Bel}\left(S_{j}\right)\right]-\max \left[0, \operatorname{Bel}\left(S_{i}\right)-\operatorname{Pls}\left(S_{j}\right)\right]}{\left[\operatorname{Pls}\left(S_{i}\right)-\operatorname{Bel}\left(S_{i}\right)\right]+\left[\operatorname{Pls}\left(S_{j}\right)-\operatorname{Bel}\left(S_{j}\right)\right]} .
$$


The group of DAs $S_{i}$ is said to be superior to $S_{j}$ (denoted by $\left.S_{i} \succ S_{j}\right)$ if $P\left(S_{i}>S_{j}\right)>0.5$. Groups of DAs $S_{i}$ and $S_{j}$ are considered indifferent (denoted by $\left.S_{i} \sim S_{j}\right)$ if $P\left(S_{i}>S_{j}\right)=0.5$.

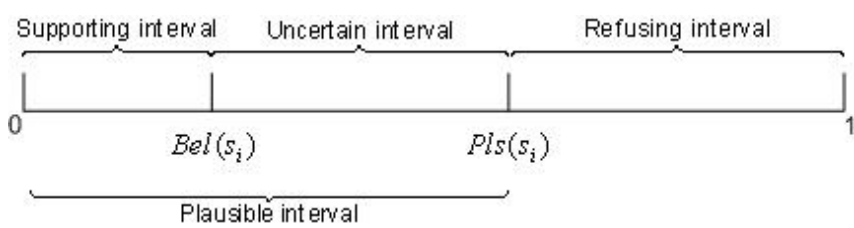

Figure 1. Interpretation of the belief interval

In this paper, three known criteria are used to test different types of rank reversal in the DS/AHP method. These criteria were applied to test other MCDM methods (Wang and Triantaphyllou, 2008).

Test criterion \#1: An effective MCDM method should not change the indication of the best DA when an irrelevant DA is added to the set of DAs (given that the relative importance of each decision criterion remains unchanged). The same should also be true for the relative rankings of the rest of the unchanged DAs.

Test criterion \#2: The rankings of DAs by an effective MCDM method should follow the transitivity property.

Test criterion \#3: For the same decision problem and when using the same MCDM method, after combining the rankings of the smaller problems that an MCDM problem is decomposed into, the new overall ranking of the DAs should be identical to the original overall ranking of the un-decomposed problem.

Let us consider the rank reversals under the Condition 1 when an irrelevant DA with different properties is added to the set of DAs.

Case 1: New DA $A_{N+1}$ is irrelevant and forms a separate group with respect to each one of the decision criteria, i.e. $\left\{A_{N+1}\right\} \cap S_{1 i}=\varnothing$ and $\left\{A_{N+1}\right\} \cap S_{2 k}=\emptyset, i=1, \ldots, D_{1}, k=1, \ldots, D_{2}$.

When such DA $A_{N+1}$ is added and the Dempster's combination rule (1) is used, the intersections' submatrix $S_{1 i} \cap S_{2 k}$ for groups of previously existing DAs $A_{1}, \ldots, A_{N}$ does not change and identified in the Table 1 in bold.

Table 1. Intermediate results of the utilization of the Dempster's combination rule (Case 1)

\begin{tabular}{|c|c|c|c|c|c|c|}
\hline$m_{1}(\cdot)$ & $S_{21}$ & $S_{22}$ & $\cdots$ & $S_{2 D_{2}}$ & $\left\{A_{N+1}\right\}$ & $\Theta$ \\
\hline$S_{11}$ & $\mathbf{S}_{11} \cap \mathbf{S}_{21}$ & $\mathbf{S}_{11} \cap \mathbf{S}_{22}$ & $\cdots$ & $\mathbf{S}_{11} \cap \mathbf{S}_{2 D_{2}}$ & $\varnothing$ & $\mathbf{S}_{11}$ \\
\hline$S_{12}$ & $\mathbf{S}_{12} \cap \mathbf{S}_{21}$ & $\mathbf{S}_{12} \cap \mathbf{S}_{22}$ & $\cdots$ & $\mathbf{S}_{12} \cap \mathbf{S}_{2 D_{2}}$ & $\varnothing$ & $\mathbf{S}_{12}$ \\
\hline$\vdots$ & $\vdots$ & $\vdots$ & $\vdots$ & $\vdots$ & $\vdots$ & $\vdots$ \\
\hline$S_{1 D_{1}}$ & $\mathbf{S}_{1 D_{1}} \cap \mathbf{S}_{21}$ & $\mathbf{S}_{1 \mathbf{D}_{1}} \cap \mathbf{S}_{22}$ & $\cdots$ & $\mathbf{S}_{1 D_{1}} \cap \mathbf{S}_{2 D_{2}}$ & $\varnothing$ & $\mathbf{S}_{1 \mathrm{D}_{1}}$ \\
\hline$\left\{A_{N+1}\right\}$ & $\varnothing$ & $\varnothing$ & $\cdots$ & $\varnothing$ & $\left\{A_{N+1}\right\}$ & $\left\{A_{N+1}\right\}$ \\
\hline$\Theta$ & $\mathbf{S}_{21}$ & $\mathbf{S}_{22}$ & $\cdots$ & $\mathbf{S}_{2 D_{2}}$ & $\left\{A_{N+1}\right\}$ & $\Theta$ \\
\hline
\end{tabular}

However, the normalization constant $K$ in the Dempster's rule (1) is changed. Therefore the aggregated mass functions for groups of DAs may be changed disproportionately when this rule is applied. Thus, rank reversals may occur. For instance, rank reversals in terms of the test criteria \#1 and \#3 are illustrated in Examples 1 and 2. 
Case 2: New DA $A_{N+1}$ is irrelevant and forms a separate group in terms of only one decision criterion. In terms of the other decision criterion this DA $A_{N+1}$ has the same measure of favorability with respect to $\Theta$ as one or several previously existing DAs, i.e. $A_{N+1}$ is included into one of the existing groups of DAs.

Let DA $A_{N+1}$ be included into the group $S_{21}$ (without loss of generality, the choice of $S_{21}$ is not detrimental). Then, after introduction of the DA $A_{N+1}$ the groups $S_{11}, S_{12}, \ldots, S_{1 D_{1}}$ and $\left\{A_{N+1}\right\}$ are identified in terms of criterion $C_{1}$ and the groups $S_{21}^{\prime}, S_{22}, \ldots, S_{2 D_{2}}$ in terms of criterion $C_{2}$, where $S_{21}^{\prime}=S_{21} \cup\left\{A_{N+1}\right\}$.

As it can be seen from the Table 2, the intersections' submatrix in the Dempster's combination rule (1) for groups of previously existing DAs (indicated in the table 2 in bold) is changed, since the intersection of group $S_{21}^{\prime}$ with the frame $\Theta$ is changed. The normalization constant $K$ in the Dempster's combination rule is also changed. Therefore, the aggregated mass functions for groups of previously existing DAs may be changed disproportionately when this rule is used. Thus, rank reversal may occur (see Example 3).

Table 2. Intermediate results of the utilization of the Dempster's combination rule (Case 2)

\begin{tabular}{|c|c|c|c|c|c|}
\hline$C_{1} C_{2}$ & $S_{21}^{\prime}=S_{21} \cup\left\{A_{N+1}\right\}$ & $S_{22}$ & $\cdots$ & $S_{2 D_{2}}$ & $\Theta$ \\
\hline$S_{11}$ & $\mathbf{S}_{11} \cap \mathbf{S}_{21}$ & $\mathbf{S}_{11} \cap \mathbf{S}_{22}$ & $\cdots$ & $\mathbf{S}_{11} \cap \mathbf{S}_{2_{2}}$ & $\mathbf{S}_{11}$ \\
\hline$S_{12}$ & $\mathbf{S}_{12} \cap \mathbf{S}_{21}$ & $\mathbf{S}_{12} \cap \mathbf{S}_{22}$ & $\cdots$ & $\mathbf{S}_{12} \cap \mathbf{S}_{2_{2}}$ & $\mathbf{S}_{12}$ \\
\hline$\vdots$ & $\vdots$ & $\vdots$ & $\vdots$ & $\vdots$ & $\vdots$ \\
\hline$S_{1 D_{1}}$ & $\mathbf{S}_{1 \mathrm{D}_{1}} \cap \mathbf{S}_{21}$ & $\mathbf{S}_{1 \mathrm{D}_{1}} \cap \mathbf{S}_{22}$ & $\cdots$ & $\mathbf{S}_{1 \mathrm{D}_{1}} \cap \mathbf{S}_{\mathbf{2 D}_{2}}$ & $\mathbf{S}_{\mathbf{1 D}_{1}}$ \\
\hline$\left\{A_{N+1}\right\}$ & $\left\{A_{N+1}\right\}$ & $\varnothing$ & $\cdots$ & $\varnothing$ & $\left\{A_{N+1}\right\}$ \\
\hline$\Theta$ & $\mathbf{S}_{21} \cup\left\{\mathbf{A}_{\mathrm{N}_{+1}}\right\}$ & $\mathbf{S}_{22}$ & $\cdots$ & $\mathbf{S}_{\mathbf{2 D}_{2}}$ & $\Theta$ \\
\hline
\end{tabular}

Let us consider the possibility of rank reversal in the DS/AHP under the test criterion \#2. Suppose that the DS/AHP method has ranked a set of DAs of a decision problem. Next, assume that this problem is decomposed into a set of smaller problems each defined on two DAs at a time and the same number of decision criteria as in the original problem. Then, according to the test criterion \#2, all the partial rankings deriving from the smaller problems should comply with the transitivity property.

Let us denote groups of DAs in terms of two decision criteria $C_{1}$ and $C_{2}$ as $S_{1 i}$ and $S_{2 j}$. Suppose each one of the groups consists of a single element, namely $S_{11}=S_{21}=\left\{A_{1}\right\}, S_{12}=S_{22}=\left\{A_{2}\right\}, \ldots$, $S_{1 N}=S_{2 N}=\left\{A_{N}\right\}$. Assume that the DAs (groups of DAs) are considered in pairs, and rankings of two arbitrary pairs are $A_{i} \succ A_{j}$ and $A_{j} \succ A_{k}$. Then the aggregated mass of DA $A_{i}$ under the Dempster's combination rule (1) is $\frac{1}{K} m_{1}\left(A_{i}\right)\left(m_{2}\left(A_{i}\right)+m_{2}(\Theta)\right)+m_{1}(\Theta) m_{2}\left(A_{i}\right)$, where $m_{1}(\cdot)$ and $m_{2}(\cdot)$ are masses with respect to decision criteria $C_{1}$ and $C_{2}, K$ is the normalization constant. Then rankings $A_{i} \succ A_{j}$ and $A_{j} \succ A_{k}$ lead to the following inequalities:

$$
\begin{aligned}
& m_{1}\left(A_{i}\right)\left(m_{2}\left(A_{i}\right)+m_{2}(\Theta)\right)+m_{1}(\Theta) m_{2}\left(A_{i}\right)-m_{1}\left(A_{j}\right)\left(m_{2}\left(A_{j}\right)+m_{2}(\Theta)\right)+m_{1}(\Theta) m_{2}\left(A_{j}\right)>0, \\
& m_{1}\left(A_{j}\right)\left(m_{2}\left(A_{j}\right)+m_{2}(\Theta)\right)+m_{1}(\Theta) m_{2}\left(A_{j}\right)-m_{1}\left(A_{k}\right)\left(m_{2}\left(A_{k}\right)+m_{2}(\Theta)\right)+m_{1}(\Theta) m_{2}\left(A_{k}\right)>0
\end{aligned}
$$

When combined, these inequalities yield: 
$m_{1}\left(A_{i}\right)\left(m_{2}\left(A_{i}\right)+m_{2}(\Theta)\right)+m_{1}(\Theta) m_{2}\left(A_{i}\right)-m_{1}\left(A_{k}\right)\left(m_{2}\left(A_{k}\right)+m_{2}(\Theta)\right)+m_{1}(\Theta) m_{2}\left(A_{k}\right)>0$, that is $A_{i} \succ A_{k}$.

Thus, if each group of DAs consists of a single element, then the transitivity property is satisfied, and, hence, rank reversal does not appear in the DS/AHP method under the test criterion \#2.

\section{Examples of rank reversals in the DS/AHP method}

Several examples are employed to illustrate different types of rank reversals in the DS/AHP method. Example 1 illustrates the Case 1 of rank reversal, when a new DA is irrelevant and forms a separate group with respect to each one of the decision criteria. In this example, the best DA is changed and, moreover, a new irrelevant DA becomes the best one. Also, in this example overall rankings of DAs from smaller problems are not identical to the original rankings before the problems' decomposition.

Example 1: Let three DAs $A_{1}, A_{2}$ and $A_{3}$ be evaluated in terms of two decision criteria $C_{1}$ and $C_{2}$ when weights of the criteria are $p_{1}=0.4$ and $p_{2}=0.6$. Three groups of DAs $\left\{A_{1}\right\},\left\{A_{2}\right\}$ and $\left\{A_{3}\right\}$ have been identified for the decision criterion $C_{1}$, and three groups of DAs $\left\{A_{1}\right\},\left\{A_{2}\right\}$ and $\left\{A_{3}\right\}$ - for the decision criterion $C_{2}$ (see Table 3).

Table 3. The knowledge matrices for the criteria $C_{1}$ and $C_{2}$ (Example 1)

\begin{tabular}{|c|c|c|c|}
\hline$C_{1}$ & $\left\{A_{1}\right\}$ & $\left\{A_{2}\right\}$ & $\left\{A_{3}\right\}$ \\
\hline$\Theta$ & $6 p_{1}$ & $8 p_{1}$ & $3 p_{1}$ \\
\hline
\end{tabular}

\begin{tabular}{|c|c|c|c|}
\hline$C_{2}$ & $\left\{A_{1}\right\}$ & $\left\{A_{2}\right\}$ & $\left\{A_{3}\right\}$ \\
\hline$\Theta$ & $4 p_{2}$ & $3 p_{2}$ & $7 p_{2}$ \\
\hline
\end{tabular}

The belief values of the groups of DAs are $\operatorname{Bel}\left(\left\{A_{1}\right\}\right)=0.3067, \operatorname{Bel}\left(\left\{A_{2}\right\}\right)=0.3143$ and $\operatorname{Bel}\left(\left\{A_{3}\right\}\right)=0.3137$. These belief values result in ranking $A_{2} \succ A_{3} \succ A_{1}$.

Suppose that a new irrelevant DA $A_{4}$ is added to the set of DAs. This DA $A_{4}$ forms the separate group with respect to each one of the decision criteria $C_{1}$ and $C_{2}$. The measures of favorability of the group $\left\{A_{4}\right\}$ with respect to the frame $\Theta$ on $C_{1}$ and $C_{2}$ are $5 p_{1}$ and $6 p_{2}$, respectively. Then, after introduction of the DA $A_{4}$, the belief values are as follows: $\operatorname{Bel}\left(\left\{A_{1}\right\}\right)=0.2215$, $\operatorname{Bel}\left(\left\{A_{2}\right\}\right)=0.2272, \operatorname{Bel}\left(\left\{A_{3}\right\}\right)=0.2284$ and $\operatorname{Bel}\left(\left\{A_{4}\right\}\right)=0.2653$. Therefore, the ranking of DAs becomes $A_{4} \succ A_{3} \succ A_{2} \succ A_{1}$. In this example, rank reversal occurs since the ranking between DAs $A_{2}$ and $A_{3}\left(A_{3} \succ A_{2}\right)$ differs from the ranking between these DAs before the DA $A_{4}$ was added $\left(A_{2} \succ A_{3}\right)$. Another type of rank reversal in this example refers to the change of the best DA, i.e. the new irrelevant $D A A_{4}$ becomes the best one.

Now suppose that the last problem with the four DAs is decomposed into the set of smaller problems. As a result, the overall ranking from the smaller problems $\left(A_{4} \succ A_{2} \succ A_{3} \succ A_{1}\right)$ is not identical to the original ranking before the problem decomposition $\left(A_{4} \succ A_{3} \succ A_{2} \succ A_{1}\right)$.

It should be noted that the decision problems described in previous Example 1, have conflicting judgements of DAs with respect to decision criteria. Rank reversals observed in these problems reflect the decision maker's rational decision making process. Let us consider the following real-life decision-making problem. Suppose two employees are evaluated in terms of two decision criteria - 
analytical ability and interpersonal skills, and these criteria are of equal importance. Suppose first employee has excellent analytical ability, but poor interpersonal skills. Second employee, on the contrary, has excellent interpersonal skills but no analytical ability. The DS/AHP method will result in equal belief measures and belief intervals for these two employees, therefore, they are of equal value. Then, a new employee is hired, who has good analytical ability, but not quite as good as the first employee, and has interpersonal skills, but not quite as good as the second employee. Since the first and second employees dominate the new employee, the new employee is irrelevant by both criteria. No doubt that these two employees should not still be more valuable than the new one, when the decision criteria are of equal importance. The new employee becomes more valuable. Rank reversal is desired in such decision making problem. The same result is obtained when the DS/AHP method is used.

The next Example 2 illustrates the Case 2 of rank reversals with the DS/AHP method when a new irrelevant DA has the same measure of favorability with respect to the frame $\Theta$ as one or several previously existing DAs, i.e. is included into one of the existing groups of DAs.

Example 2: Consider the simplified example 1 of the choice of textbooks (Beynon, 2005a) when ten DAs A - $\mathrm{J}$ are evaluated in terms of two decision criteria $C_{1}$ and $C_{3}$ with a positive CPVs 0.4 and 0.6 respectively. Suppose that a new irrelevant DA $K$ is added to the set of these DAs. It has the same measure of favorability with respect to the frame $\Theta$ as DA $J$ on the decision criteria $C_{1}$, and forms the separate group on the decision criteria $C_{3}$. If the measures of favorability of the groups $\{J, K\}$ and $\{K\}$ on $C_{1}$ and $C_{3}$ are $p_{1}$ and $p_{3}$ respectively, then, the bpa values for the groups of DAs and the frame $\Theta$ in terms of criteria $C_{1}$ and $C_{2}$ are as follows: $m_{1}(\{F\})=0.3333, m_{1}(\{A, H\})=0.2222$, $m_{1}(\{C, D, I\})=0.1111, m_{1}(\{J, K\})=0.0556, m_{1}(\Theta)=0.2778, m_{3}(\{E, F\})=0.3488, m_{3}(\{A, G, H\}$ )$=0.2093, m_{3}(\{B, C, J\})=0.1395, m_{3}(\{K\})=0.0698$ and $m_{3}(\Theta)=0.2326$.

Table 4. The aggregated masses $m_{\text {aggr }}=m_{1} \oplus m_{3}$ and belief values $\mathrm{Bel}$ after combining all evidences

\begin{tabular}{|c|c|c|c|c|c|c|c|c|c|c|c|}
\hline & $\{C\}$ & $\{F\}$ & $\{J\}$ & $\{K\}$ & $\{A, H\}$ & $\{E, F\}$ & $\{J, K\}$ & $\{A, G, H\}$ & $\{B, C, J\}$ & $\{C, D, I\}$ & $\Theta$ \\
\hline$m_{a g g r}$ & $\begin{array}{c}0.024 \\
4\end{array}$ & $\begin{array}{c}0.304 \\
8\end{array}$ & $\begin{array}{c}0.012 \\
3\end{array}$ & $\begin{array}{c}0.036 \\
6\end{array}$ & 0.1545 & 0.1524 & 0.0203 & 0.0915 & 0.0610 & 0.0406 & $\begin{array}{c}0.101 \\
6\end{array}$ \\
\hline Bel & $\begin{array}{c}0.024 \\
4\end{array}$ & $\begin{array}{c}0.304 \\
8\end{array}$ & $\begin{array}{c}0.012 \\
3\end{array}$ & $\begin{array}{c}0.036 \\
6\end{array}$ & 0.1545 & 0.4572 & 0.0692 & 0.2460 & 0.0977 & 0.0650 & $\begin{array}{c}1.000 \\
0\end{array}$ \\
\hline
\end{tabular}

The belief-based ranking order of DAs $C$ and $J$ is $C \succ J$ (see Table 4). As a result, the rank reversal occurs under Condition 1, since ranking order of these DAs was $J \succ C$ (see Section 3) before introduction of the DA $K$.

\section{Conclusions}

This paper describes different cases of rank reversals in the DS/AHP method. These cases deal with the rank reversals when an irrelevant DA - a DA that is dominated by one or more previously existing DAs - is added or removed. The results indicate that the rank reversals may appear in case of adding an irrelevant DA which forms a separate group with respect to each one of the decision criteria when the DS/AHP method is used to solve decision problems with conflicting judgements of DAs in terms of the criteria. Therefore, such rank reversals reflect the decision maker's rational decision making process.

There are real-life decision problems where the rank reversal is allowed and the problems where this property must be precluded. For instance, rank reversal is allowed in problems with fixed resources. However, in decision problems when resources can be added of removed rank reversal must not 
occur. It is the very first time that rank reversals are reported to occur in the DS/AHP method. Thus, this phenomenon requires additional investigation, and more intensive study should be conducted to find rates of rank reversals on the indication of the best and any DAs.

\section{REFERENCES}

Barzilai, J., Lootsma, F.A. (1994). Power Relations and Group Aggregation in Multiplicative AHP and SMART. Proceedings of the 3rd International Symposium on The Analytic Hierarchy Process. Washington, DC, 157-168.

Beynon, M.J., Curry, B., Morgan, P.H. (2000). The Dempster-Shafer theory of evidence: an alternative approach to multicriteria decision modeling. Omega 28 (1), $37-50$.

Beynon, M. J. (2002). DS/AHP method: A mathematical analysis, including an understanding of uncertainty. European Journal of Operational Research 140, 148-164.

Beynon, M. J. (2005). A method of aggregation in DS/AHP for group decision-making with the nonequivalent importance of individuals in the group. Computers \& Operations Research 32, 1881-1896.

Dyer, J.S. (1990). Remarks on the analytic hierarchy process. Management Science 36, $249-258$.

Forman, E.H. (1987). Relative vs Absolute Worth. Mathematical Modelling 9(3-5), 195-202.

Fortes, I., Mora-L'opez, L., Morales, R., Triguero, F (2006). Inductive learning models with missing values. Mathematical and Computer Modelling 44, 790 - 806.

Hua, Z., Gong, B., Xu, X. (2008). A DS-AHP approach for multi-attribute decision making problem with incomplete information. Expert Systems with Applications 34, 2221-2227.

Luce, R.D., Raiffa, H. (1957). Games and Decisions. John Wiley and Sons, Inc., New York.

Nedashkovskaya, N. I. (2005). Ranking reversals when the AHP method is used. System Research and Information Technologies 4, 120 - 130. (In Ukrainian)

Nedashkovskaya, N.I. (2010). Multiple-criteria decision making under incomlete expert judgements using the analytic hierarchy process (AHP) and the Dempster-Shafer theory. Journal of the Mykholaiv Petro Mogyla State University: Computer Technologies Series 143, 6 - 14. (In Russian)

Quinten, A., Raaijmakers, W. (1999). Effectiveness of different missing data treatments in surveys with Likert-type data: Introducing the relative mean substitution approach. Educational and Psychological Measurement 59 (5), 725 - 748.

Saaty, T.L. (1988). The Analytic Hierarchy Process. RWS Publication, Pittsburg, PA.

Saaty, T.L. (1990). An Exposition of the AHP in Reply to the Paper "Remarks on the Analytic Hierarchy Process". Management Science 36 (3), 259-268.

Saaty, T.L. (2003). Decision-making with the AHP: Why is the principal eigenvector necessary. European Journal of Operational Research 145(1), 85 - 91.

Saaty, T.L. (2006). Rank from comparisons and from ratings in the analytic hierarchy/ network processes. European Journal of Operational Research 168(2), 557 - 570. 
Triantaphyllou, E. (2001). Two New Cases of Rank Reversals when the AHP and Some of its Additive Variants are Used that do not Occur with the Multiplicative AHP. Journal of Multi-Criteria Decision Analysis 10(1), 11-25.

Wang Y.-M., Yang J.-B., Xu D.-L., Chin K.-S. (2006). The evidential reasoning approach for multiple attribute decision analysis using interval belief degree. European Journal of Operational Research 175(1), 35 - 66 .

Wang, X., Triantaphyllou, E. (2008). Ranking irregularities when evaluating alternatives by using some ELECTRE methods. Omega 36 (1), 45 - 63. 